\title{
Grating speckle method for in-plane displacement measurement
}

\author{
Meirong Tu, James McKelvie, Fu-long Dai, and Daniel Post
}

\begin{abstract}
A high sensitivity technique combining lensless speckle photography and a grid technique is proposed and demonstrated. A high frequency grid is applied to the specimen surface anci contact specklegrams are made in white light before and after loading, in the double exposure mode. Subsequent optical processing yields the various components of in-plane displacement. The sensitivity can be chosen equivalent to the grid frequency or multiples thereof. The method is simple and gives low-noise fringe patterns with high contrast. It is suitable for application in the mechanical laboratory and field environments, because vibration isolation precautions are not necessary.
\end{abstract}

\section{Introduction}

In the so-called close range objective speckle meth$\operatorname{od}^{1,2}$ the speckles are produced intrinsically from a scattering surface and recorded directly on a photosensitive material without the use of lenses. The spectral range inherent in this double exposed specklegram is usually much higher than that achieved using a lens. By means of this method we can obtain whole-field fringe patterns of in-plane displacements. A double exposed holographic plate placed in the optical Fourier Transform system indicated in Fig. 1 has its spectral composition displayed in the spatial frequency plane $M$. The display contains contributions equivalent to those from a large number of gratings. When filtering takes place, all other components contribute zero to the image plane except those that pass through the aperture, which are characterized by a particular mean frequency. Accordingly, the fringe pattern that appears on the image plane is similar to a moire fringe pattern. The mean frequency is determined by

$$
\nu_{m}=\frac{\sin \theta}{\lambda} \text {. }
$$

M. Tu is from Chinese Academy of Sciences, Institute of Mechanics, Beijing, China; her current address is Florida State University, Department of Mechanical Engineering, Tallahassee, Florida 32316. J. McKelvie is with University of Strathclyde, Department of Mechanics of Materials, Glasgow G11XL; U.K. F. Dai is with Tsinghua University, Department of Engineering Mechanics, Beijing, China. D. Post is with Virginia Polytechnic Institute State University, Department Engineering Science Mechanics, Blacksburg, Virginia 24061.

Received 18 January 1989.

0003-6935/89/163354-04\$02.00/0.

(C) 1989 Optical Society of America.
However, only a little of the total available energy (namely, that related to a narrow band of frequency) has been utilized to form the image pattern during the reconstruction. It is generally insufficient when high sensitivity fringe patterns are desired, since typical surfaces diffract relatively weakly to high angles. Also the signal-to-noise ratio is poor; it is desirable to keep the aperture small to increase the contrast, but reducing the aperture increases the speckle size and the optical noise.

In this work, the concept of using a regular grating instead of a randomly scattering surface is proposed with the following rationale. Since the energy would be concentrated near harmonically related frequencies the process ought to yield fringe patterns of superior quality, with a sensitivity corresponding to the grating frequency. Yet, the advantages of the close-range objective speckle technique would be retained.

\section{Basic theory}

Since a periodic function is associated with a discrete spectrum, the energy distribution in the frequency plane will, in principle, be concentrated at a few points. Let a high frequency grating be replicated onto the specimen surface, and let white light objective speckle recordings be made before and after loading, as illustrated in Fig. 2. Then the double-exposed specklegram will encode the Fourier components of the surface, as represented by the diffracted beams, for the undeformed and deformed conditions. During the reconstruction procedure illustrated in Fig. 3, we can isolate the components and get the corresponding fringe pattern.

An object may be either self-luminous or irradiated by some incident wave field. In either case, we regard the object as a collection of independent point radiators, the strength of which varies from point to point 


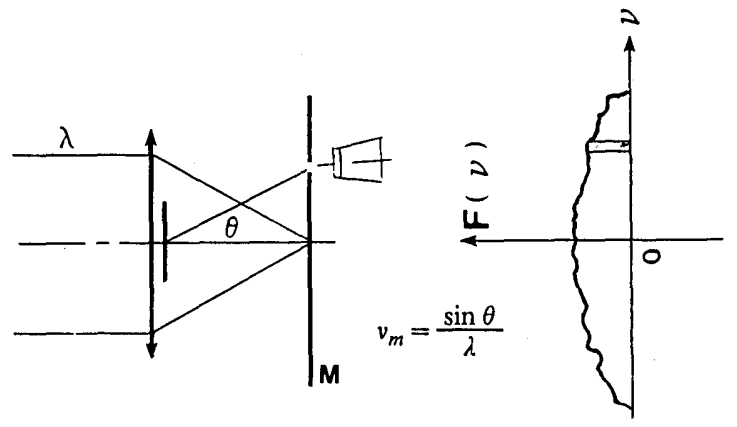

Fig. 1. Reconstruction of specklegram.

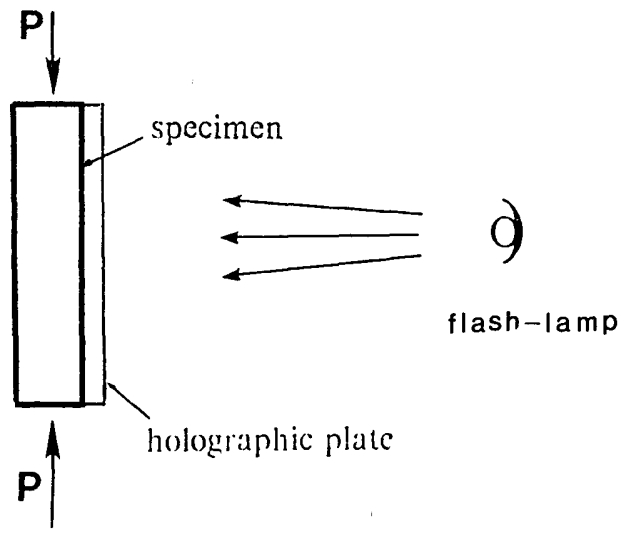

Fig. 2. Recording system.

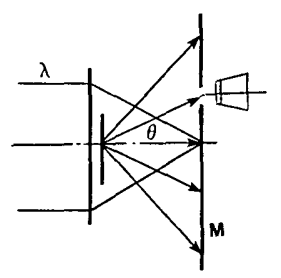

(a)

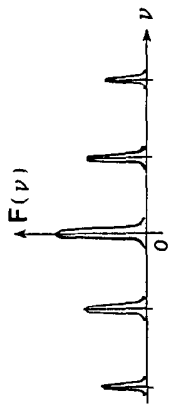

(b)

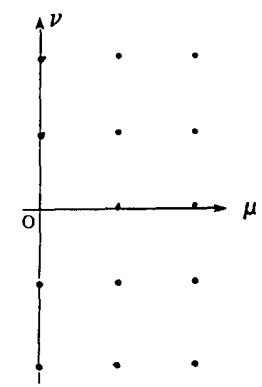

(c)
Fig. 3. Grating specklegram reconstruction: (a) reconstruction procedure; (b) schematic of energy distribution; (c) appearance in the $M$-plane for orthogonal gratings.

according to some information-bearing characteristic of the object, e.g., its reflectance, diffuseness, and finest scale of optical structure. Changes in this radiance distribution occur as the surface deforms, and thus the radiance can be used to determine the displacement.

A speckle photograph, on the other hand, involves an image-forming system which maps the radiance distribution of the object as some irradiance distribution at the holographic plate. The mapping involves the coherent optical transfer function of the imaging system, which will always involve a cutoff frequency. (Even the observable radiance itself acts as a low pass

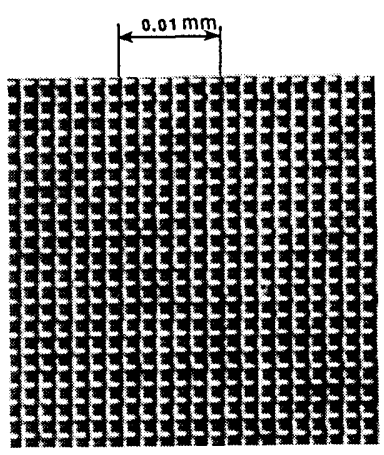

(a)

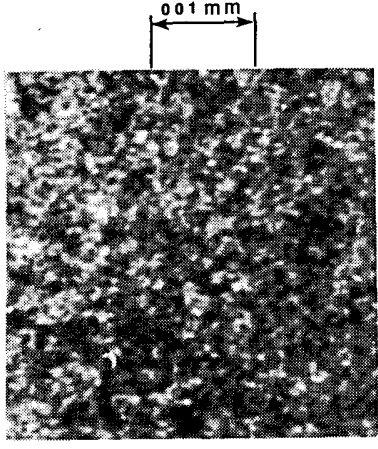

(b)
Fig. 4. (a) Enlargement of specimen grid. (b) Enlargement of corresponding contact specklegram (same magnification, single exposure).

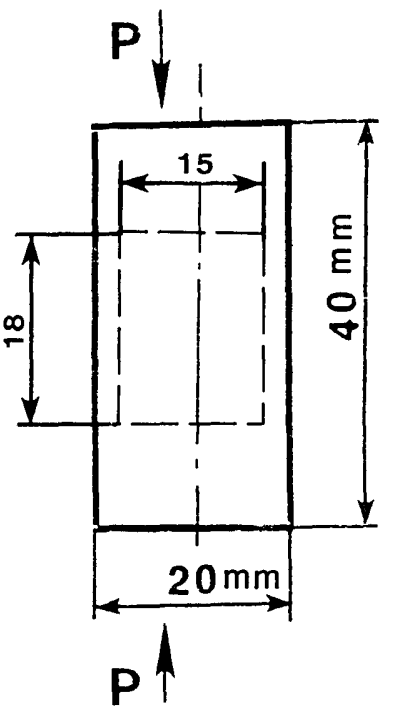

Fig. 5. Specimen and loading geometries.

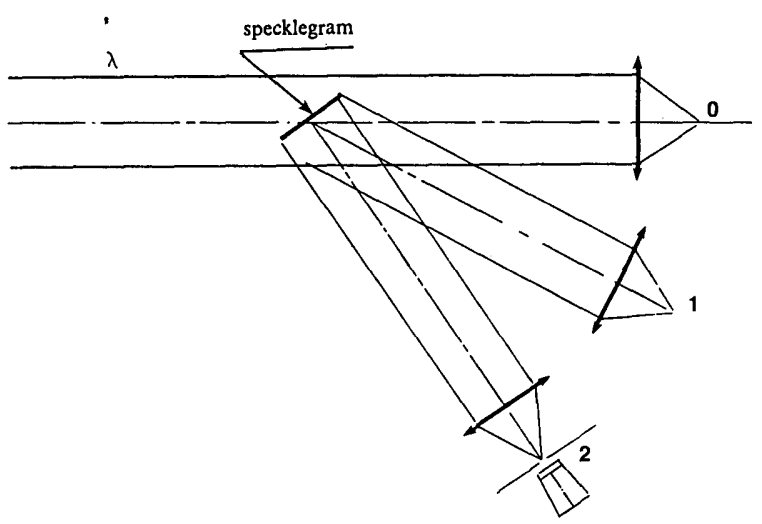

Fig. 6. Reconstruction system for grating specklegram.

filter, since variations having spatial frequency higher than $1 / \lambda$ cannot propagate. ${ }^{3,4}$ )

A lensless recording system can in principle receive almost all the Fourier components that the surface exhibits, within the above limitation, provided the re- 

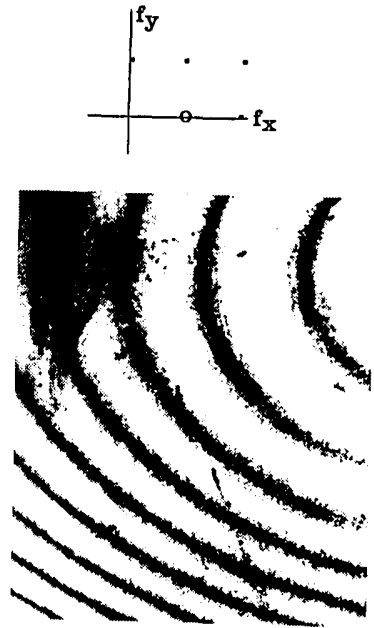

a) $f_{\mathrm{x}}=6001 / \mathrm{mm}$ $f_{y}=0$
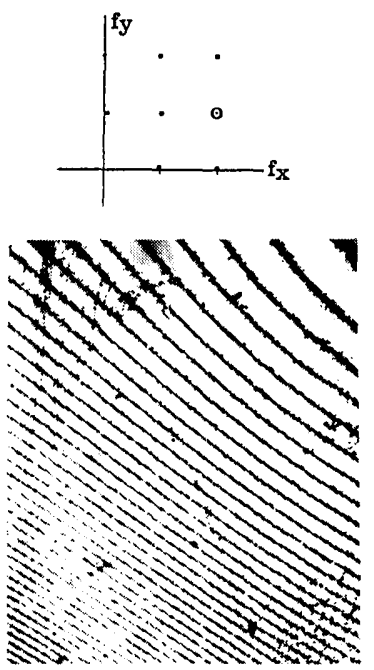

e)
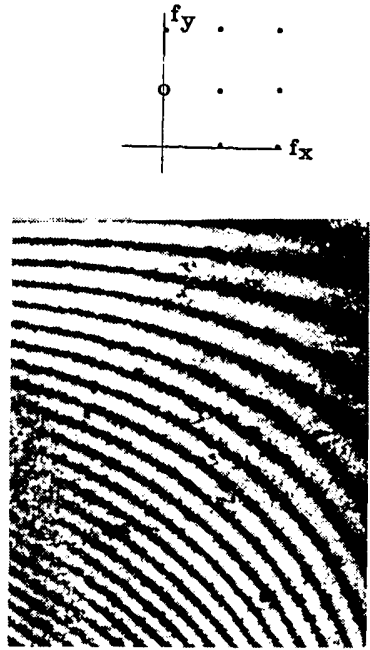

b) $f_{x}=0$

$f_{\bar{y}}=6001 / \mathrm{mm}$
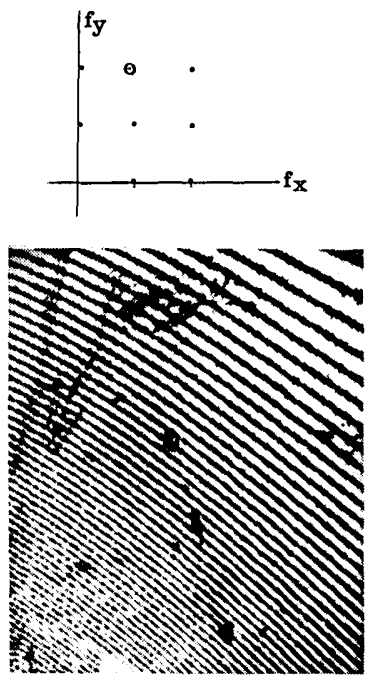

f)

$\mathrm{f}_{\mathrm{X}}=600 \mathrm{l} \mathrm{l} / \mathrm{mm}$ $f_{y}=1200 \quad 1 / \mathrm{mm}$
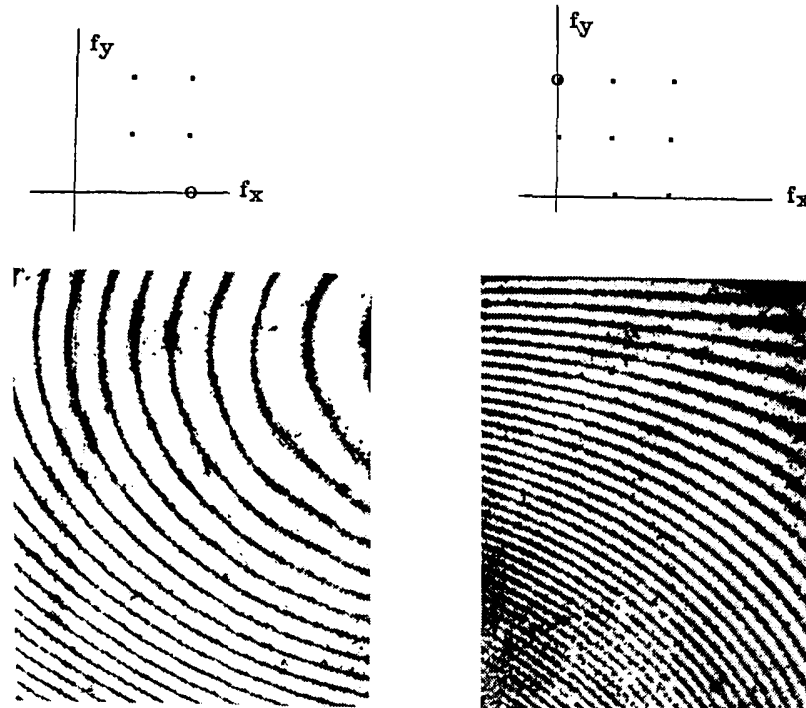

c) $f_{X}=12001 / \mathrm{mm}$ $f_{y}=0$

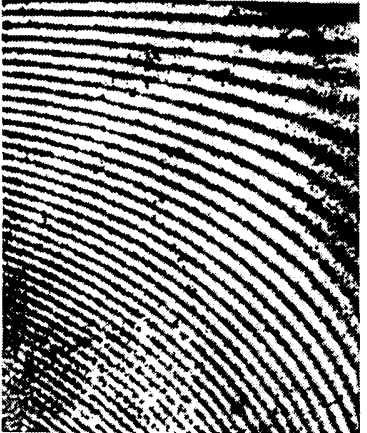

d) $\mathbf{f}_{\mathbf{X}}=0$

$f_{y}=1200 \quad 1 / \mathrm{mm}$
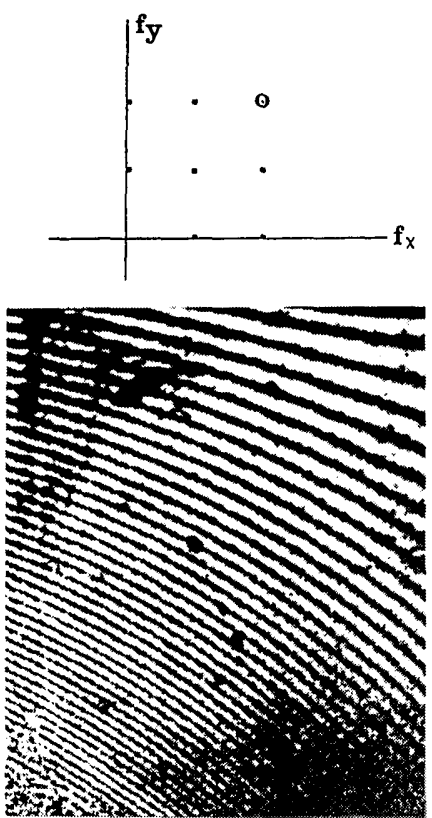

g) $f_{\mathrm{X}}=1200 \mathrm{l} / \mathrm{mm}$

$f_{y}=1200 \mathrm{l} / \mathrm{mm}$

Fig. 7. Fringe patterns of in plane displacements obtained from different locations of the filtering aperture.

cording material is large enough. As the light propagates, relative phase changes occur between the various frequency components and, unlike a focused lens system, there is in general no point-to-point correspondence between the received irradiance and the surface radiance. In the case where the recording material is in contact with the radiating surface, however, there is virtual point-to-point correspondence. Such a configuration also lends itself to the use of white light, since the coherence length needed is then only a few micrometers. As shown in Fig. 4, the speckle pattern obtained from a regular grid does not correspond in any obvious way to the structure of the grid. We may imagine the speckle pattern arising out of the incoherent addition of myriad regular interference patterns, with random relative phase relationships. Nevertheless the speckle pattern is an encoding of the surface radiance and can be interrogated to yield displacement information.

Figures 4(a) and (b) show optical structures corre- 
sponding to the object surface and the corresponding specklegram at the same magnification. On the object surface there is a crossed line phase grating with a fundamental frequency of 600 lines $/ \mathrm{mm}$. After an exposure using a white light source, the holographic plate displays a very irregular optical structure resembling a typical conventional specklegram pattern. Despite the marked difference, however, the frequencies encoded in both are almost the same.

\section{Experimental Validation}

A specimen made of epoxy was loaded in asymmetric compression as shown in Fig. 5. A high frequency crossed line phase grating had previously been replicated on the specimen surface, the fundamental frequency being 600 lines $/ \mathrm{mm}$ in both the $x$ and $y$ directions. The recording system was that shown in Fig. 2. A holographic plate was clamped lightly to the specimen surface, with the emulsion layer against the specimen. A photographic flashlamp was used as the light source, the specimen being illuminated through the plate. Having been exposed before and after loading, the holographic plate (after development) was placed in the reconstruction system shown in Fig. 6. This system is equivalent to that of Fig. 3 but in the case of large diffraction angles, it is easier to maintain the specimen plane and image plane parallel to each other. (The example illustrated is the geometry for interrogation using the second diffraction order. The angle of the specklegram is appropriately altered for other cases.) Seven different fringe patterns are shown in Fig. 7, each obtained after filtering in different Fourier plane positions. The position and corresponding frequencies $\left(f_{x}, f_{y}\right)$ are indicated for each pattern. The patterns represent the $15-\times 18-\mathrm{mm}$ area shown by dashed lines in Fig. 5.
The pattern of Fig. 7(g) with a frequency of 1200 lines/mm in both the $x$ and $y$ directions, can be considered having a frequency of 1697 lines $/ \mathrm{mm}$ in the $45^{\circ}$ direction. The quality of fringes in all cases is seen to be markedly superior to those illustrated in, for example, Ref. 1. The correspondence of the sensitivity (as determined by the aperture position) with the fringe pattern is quite obvious.

\section{Conclusion}

The combination of the objective white light speckle method with grid technology is a practical extension of both moire and speckle methods. It has been demonstrated at a sensitivity corresponding to moire with 1697 lines $/ \mathrm{mm}$. The resulting fringes exhibit good fringe contrast and low speckle noise level. The effective and very simple recording system offers the possibility of application in mechanical laboratory and field environments for practical measurements relating to engineering design and verification. Stringent vibration isolation requirements are unnecessary. The technique combines most advantages of moire interferometry and the speckle method, but real time observation is not achieved.

\section{References}

1. Tu Meirong, Li Minhua and Han Jinhu, "Strain Determination on Curved Surfaces by Objective White Light Speckles," Proc. Soc. Photo-Opt. Instrum. Eng. 814, 167-172 (1987).

2. P. M. Boone, "Use of Reflection Hologram in Holographic Interferometry and Speckle Correlation for Measurement of SurfaceDisplacement," Opt. Acta 22, 579-589 (1975).

3. J. D. Gaskill, Linear Systems, Fourier Transform and Optics (Wiley, New York, 1978).

4. J. W. Goodman, Introduction to Fourier Optics (McGraw-Hill, New York, 1968).

Support for this work from the National Center for Composite Materials Research under ONR contract N00014-86-K0799, from the Chinese National Scientific Fund Committee, and from the U.S. National Academy of Sciences under the exchange program of the Committee on Scholarly Communication with the People's Republic of China, is gratefully acknowledged.

The work was performed at Virginia Polytechnic Institute and State University. 